\title{
UV-VISIBLE AND NEAR IR ABSORPTION CHARACTERISTICS OF INTERSTELLAR PAHS. I. $\mathrm{C}_{10} \mathrm{H}_{8}{ }^{+}$
}

\author{
F. SALAMA and LJ. ALLAMANDOLA \\ NASA-Ames Research Center. Space Science Division, MS: 245-6 \\ Moffett Field, CA 94035, U.S.A
}

\begin{abstract}
We have initiated a systematic and detailed study of the spectroscopy of neutral and ionized Polycyclic Aromatic Hydrocarbons (PAHs). We report here, the results obtained for the smallest PAH $\left(\mathrm{C}_{10} \mathrm{H}_{8}\right)[1]$ and discuss their astrophysical applications.
\end{abstract}

\section{Introduction}

Polycyclic Aromatic Hydrocarbons (PAHs) in their neutral and ionized states have been postulated as an important, ubiquitous component of the interstellar material. They are considered as the best candidates for the carriers of the UIR features, and they may also play a role in other critical astrophysical issues such as: (i) The FUV part of the interstellar extinction curve and the $2200 \AA$ hump. (ii) The carriers of the diffuse interstellar bands DIBs. (iii) The radiation and energy balance in space (UV to IR conversion). (iv) The extended red emission (ERE) seen in reflection nebulae. There is therefore a crucial need for quantitative UV-Visible-NIR spectroscopic data on isolated, neutral and ionized PAHs to test the existing theories and their implications.

\section{Experimental Approach and Results:}

A computer-controlled UV-Visible-NIR spectrometer system, coupled to a cryogenic cell, was designed to measure the absorption and emission spectra of neutral, ionic, or radical species in the $1800-9000 \AA$ range under conditions relevant to astrophysical environments. Matrix Isolation Spectroscopy is particularly well adapted here because it involves the trapping of the species of interest in a chemically inert, rigid cage at low temperature. 2.1: Neutral naphthalene $\left(C_{10} H_{8}\right)$ : The UV-Visible absorption spectra of $\mathrm{C}_{10} \mathrm{H}_{8}$ isolated in $\mathrm{Ar}$ and Ne matrices [1] indicate that neutral naphthalene does not absorb in the visual and therefore cannot contribute to the DIBs. The UV spectrum of naphthalene consists of three systems peaking at 3115,2792 , and $2116 \AA$ respectively in neon. The entire spectrum is dominated by a very strong band at $2116 \AA$ in Ne and $2162 \AA$ in Ar. The Ne matrix results show that the strongest absorption of $\mathrm{C}_{10} \mathrm{H}_{8}$ does not peak at the interstellar $2175 \AA$ absorption but, if present, can contribute as substructure to the hump of the extinction curve. The cross section of the $2116 \AA$ band $\left(410^{-16} \mathrm{~cm}^{2} / \mathrm{molecule}\right)$, requires a $\mathrm{C}_{10} \mathrm{H}_{8}$ abundance of $1.310^{-7}$ with respect to hydrogen in order to be detectable as substructure on the $2200 \AA$ hump (at the $10 \%$ level). The effect of the matrix material on the band positions has been studied [1] and it has been shown that it is mandatory to utilize neon as the matrix material for astrophysical applications ( $\mathrm{Ne}$ is the closest to the gas phase conditions). 
2.2: Ionized naphthalene: The UV-Visible absorption spectrum of $\mathrm{C}_{10} \mathrm{H}_{8}{ }^{+}$, formed by direct photoionization of the neutral molecule isolated in a Ne matrix, can be separated into two components (Fig. 1): the discrete features and the continuum. The spectrum indicates that contrary to its neutral precursor, $\mathrm{C}_{10} \mathrm{H}_{8}{ }^{+}$does absorb in the visual, raising a possible connection with the DIBs. The small number of DIBs $(\sim 15 \%)$ which could be accounted for by $\mathrm{C}_{10} \mathrm{H}_{8}{ }^{+}$shows, however, that $\mathrm{C}_{10} \mathrm{H}_{8}{ }^{+}$alone can not explain the DIBs in this wavelength range. This comparison hints, however, to the fact that a family of different PAH ions may contribute to the DIBs. The answer to this puzzling problem can only be obtained through a systematic comparison of the DIBs with appropriate laboratory data. The complete UVVisible spectrum (not shown) of the naphthalene cation consists of 7 systems with different intensities. Another striking aspect of these experiments is the production of a broad and strong CONTINUUM extending from the UV to the visible (2000 - 5100 $\mathrm{A})$. The laboratory spectra indicate that the growth of the cation discrete absorption features and continuum under VUV irradiation is correlated to the depletion of the neutral precursor absorption. Because of its potential astrophysical importance, it was necessary to better determine the carrier of this continuous feature. A number of experiments [1,2] which were carried out to eliminate other possibilities (the anionic counterpart $\mathrm{C}_{10} \mathrm{H}_{8}{ }^{-}$, or a dissociative transition within the neutral precursor) showed that the discrete bands due to $\mathrm{C}_{10} \mathrm{H}_{8}{ }^{+}$and the continuum grow together as a function of VUV irradiation time indicating that $\mathrm{C}_{10} \mathrm{H}_{8}{ }^{+}$is most likely responsible for the continuum. Such a broad, strong continuum has the potential to be very significant for astrophysics. If further laboratory experiments establish that this continuum is indeed a general characteristic of ionized PAHs, this may be the channel through which interstellar $U V$-Visible radiation is converted to the discrete IR emission bands.

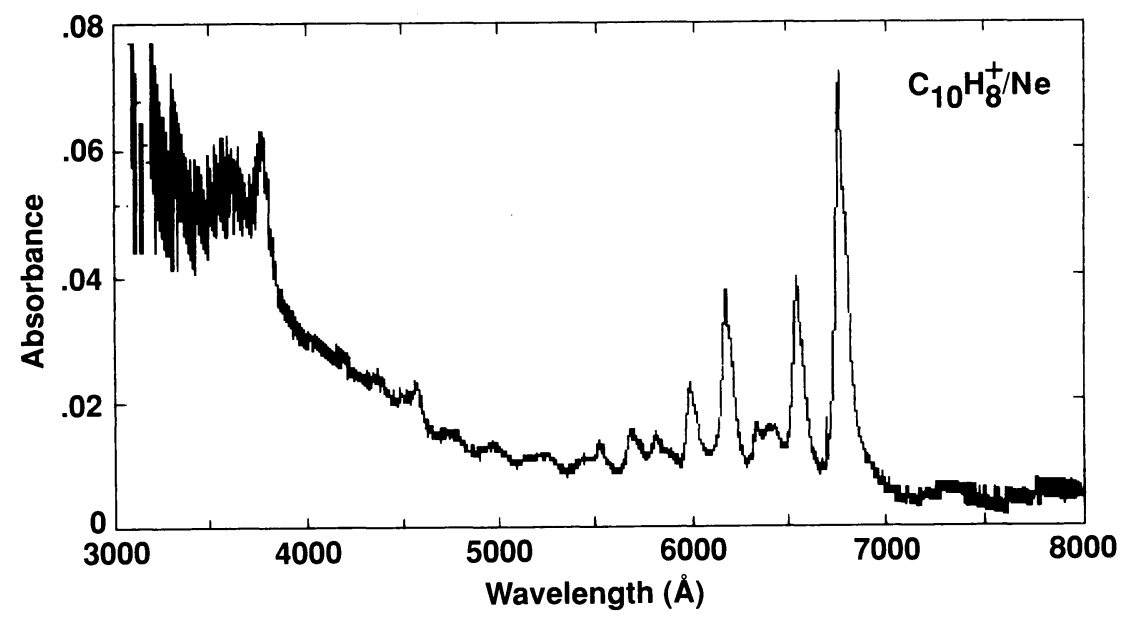

Figure 1: The absorption spectrum of $\mathrm{C}_{10} \mathrm{H}_{8}+$ in a $\mathrm{Ne}$ matrix. Experimental conditions: $\mathrm{C}_{10} \mathrm{H}_{8} / \mathrm{Ne}=1: 600,4.2 \mathrm{~K}, 20 \mathrm{~min}$ VUV irradiation with $10.2 \mathrm{eV}$ photons.

\section{References:}

1- Salama, F. and Allamandola, L. J. (1991) 'Electronic absorption spectroscopy of matrix -isolated polycyclic aromatic hydrocarbon cations. I. The naphthalene cation $\left(\mathrm{C}_{10 \mathrm{H}_{8}}{ }^{\prime}\right)^{\prime}$, J. Chem. Phys. 94 (11), 6964-6977.

2- Salama, F. and Allamandola, L. J. (1991) 'The role of matrix material and $\mathrm{CCl}_{4}$ (electron acceptor) on the ionization mechanisms of matrix-isolated naphthalene', J. Chem. Phys. (in press, Oct. 15 edition). 